\title{
Application of Extension Neural Network Algorithm and Empirical Mode Decomposition Method to Partial Discharge Diagnosis of Power Capacitors
}

\author{
Meng-Hui Wang, Shiue-Der Lu, Kun-De Lin, and Chian-Yi Pan \\ Department of Electrical Engineering, National Chin-Yi University of Technology, \\ No. 57, Sec. 2, Zhongshan Rd., Taiping Dist., Taichung City 411, Taiwan (R.O.C.)
}

(Received March 20, 2019; accepted September 9, 2019)

Keywords: power capacitor, extension neural network, chaos theory, empirical mode decomposition, chaos synchronization detection method

Power capacitors are extensively used in power systems; thus, any internal capacitor fault can affect their safe operation. The most common faults include dampness, partial discharge, aging or insulation deterioration, and structural deterioration. The purpose of this study is to use a human-machine interface diagnosis system to detect the type of power capacitor fault, so as to determine the real-time status of the power capacitor. Partial discharge data are measured and diagnosed for the power capacitors that are functional and remaining in long-term highvoltage operation. The defects are handled before the capacitor is measured by a high-frequency current transformer (HFCT) sensor, and one power platform tester is used to measure partial discharge in the capacitor case. The voltage is increased until the partial discharge phenomenon stops, and the voltage and partial discharge signals are visualized by using a high-frequency oscillograph. Afterwards, the feature of the discharge signal is determined by the empirical mode decomposition (EMD) method, combined with the chaos synchronization detection analysis method to establish the chaotic error scatter map of the discharge voltage. Then, the chaos eyes are used as the features of fault diagnosis, and the extension neural network (ENN) algorithm is used for capacitor fault recognition. The advantages of this method are that the feature extraction data volume can be reduced, subtle changes in power capacitor discharge voltage signal can be detected effectively so as to detect the power capacitor's operating state, and emergency measures can be executed in advance to prevent severe disasters. The proposed method is validated by actual measurements. The detection rate of the ENN fault detection method is $95 \%$, proving that this method is applicable to power capacitor partial discharge detection.

\section{Introduction}

In recent years, with the rapid development of power systems, the requirement for power quality has been growing gradually. Whether the power system can remain in a safe and stable *Corresponding author: e-mail: sd1@ncut.edu.tw https://doi.org/10.18494/SAM.2019.2380 
operating state or not affects national economic development and people's quality of life. When the power system remains in a high-temperature, high-voltage, and high-load state for a long time, the equipment life may fail with time. If power capacitors can be replaced and repaired before the occurrence of a fault, the operating cost during internal capacitor shutdown can be reduced greatly, thereby saving power cost from economic and environmental perspectives. ${ }^{(1)}$ Therefore, the partial discharge diagnosis of power capacitors will be the key point of this study.

Research reports on power capacitors indicate that common faults include insulating oil spillage, insulation aging, dampness, holder discharge, connection strap dropout, and immersion defectiveness. ${ }^{(2-5)}$ Over the last decade, a number of studies have been performed on the failure detection of power capacitors. ${ }^{(6-9)}$ Sharma et al. proposed a fault detection strategy considering single-end-measured fault current data for series capacitors compensating for three-phase transmission lines by using fast Walsh-Hadamard transform (FWHT). ${ }^{(6)}$ Jouybari-Moghaddam et al. used an indicating quantity superimposed reactance (SR) method to rapidly locate and identify capacitor element failures in shunt capacitor banks. ${ }^{(7)}$ Hannonen et al. utilized an output voltage transient analysis method to detect capacitor degradation under various operating conditions at the output stage of a step-down dc-dc converter. $^{(8)}$ Carvalho et al. presented a virtual instrumentation system developed through acoustic partial discharge signal detection and capacitance monitoring for the assessment of high-voltage-power capacitors. ${ }^{(9)}$

The aforementioned fault types are discussed in this paper. A defective power capacitor is made first, and the electric power testing machine increases the voltage for partial discharge testing. When the insulation of the capacitor is defective or aged, the discharge pulse damages the insulating layer inside the capacitor, inducing penetrating destruction. Afterwards, the power capacitor's terminal-to-ground partial discharge signals are measured using a highfrequency current transformer (HFCT) sensor. The fault feature is extracted by empirical mode decomposition (EMD) with the chaos theory for chaos synchronization analysis. ${ }^{(10)}$ Finally, the extension neural network (ENN), ${ }^{(11,12)}$ EMD, and the chaos method are integrated with hardware circuits and sensors to implement a human-machine interface fault diagnosis system designed with LabVIEW. The results show that the proposed method has the best recognition results compared with the multilayer neural network ${ }^{(13)}$ and extension method, ${ }^{(14)}$ and the large amount of data could be decreased greatly by EMD and chaos synchronization detection.

In Sect. 2, the power capacitor partial discharge testing results will be given. The proposed fault diagnosis algorithms, including EMD, the chaos theory, and ENN, will be introduced in Sect. 3. Signal measurement and results will be discussed in Sect. 4. Finally, conclusions will be given in Sect. 5 .

\section{Power Capacitor's Partial Discharge Test}

The partial discharge pulse signal of the power capacitor is measured in this study. Owing to the long cycle and high frequency of the extracted discharge signal, the data volume is very large. 
There may be too many interference factors during the test, so it is difficult to identify the noise in a partial discharge signal. In order to capture the high-frequency signal in the partial discharge signal, the low-frequency signal and noise are filtered out using our original high-pass filter circuit to extract the required high-frequency signal. ${ }^{(15,16)}$

\subsection{Power capacitor defect construction}

We use the internal capacitor form, the Shihlin Electric Machinery Co., Ltd., 440 V/50 KVAR power capacitor bank, as the object of partial discharge measurements, including normal and aged capacitor measurements, as shown in Fig. 1. The specifications are shown in Table 1. The test results show that long-term high temperatures inside the capacitor causes aging, as shown in Fig. 2.

\subsection{Power capacitor measurement procedure}

We use an $\mathrm{AC}$ voltage of $220 \mathrm{~V}$ to test the insulating property of the power capacitor. The single-phase, terminal-to-ground system is boosted by the power electric testing machine. The HFCT sensor is connected to the ground terminal, and the signal is received by the oscillograph and sent via USB transfer to the computer for signal analysis, as shown in Fig. 3. ${ }^{(17)}$ When the insulation of the single-phase capacitor is boosted to the critical point, the discharge pulse breaks down the insulating layer to induce failure, the pulse flows to the ground terminal by means of the transport property, and the discharge pulse is coupled with the HFCT sensor. ${ }^{(18)}$

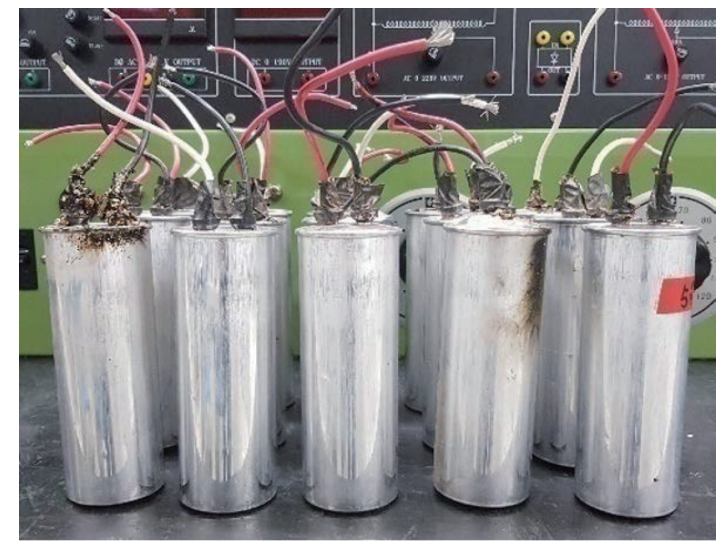

Fig. 1. (Color online) Normal and aged power capacitors.

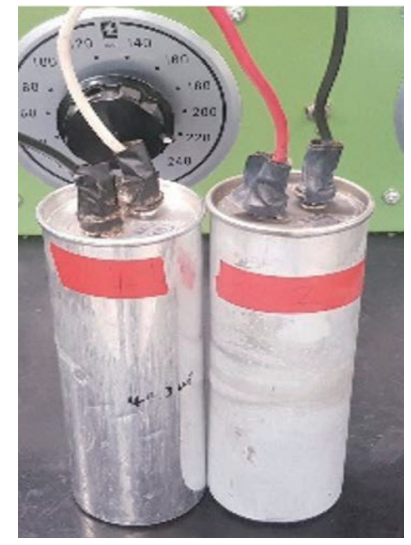

Fig. 2. (Color online) Aged power capacitors.

Table 1

Specifications of power capacitor.

\begin{tabular}{lcccc}
\hline \multicolumn{4}{c}{ Single-phase power capacitor specification } \\
\hline Capacitor state & Normal & Aged $20 \%$ & Aged $40 \%$ & Aged $60 \%$ \\
Capacitor value & $45 \mu \mathrm{F}$ & $36 \mu \mathrm{F}$ & $27 \mu \mathrm{F}$ & $18 \mu \mathrm{F}$ \\
Phase & \multicolumn{4}{c}{ Single phase } \\
Frequency & \multicolumn{2}{c}{$60 \mathrm{~Hz}$} \\
Rated voltage & \multicolumn{4}{c}{$440 \mathrm{~V}$} \\
\hline
\end{tabular}




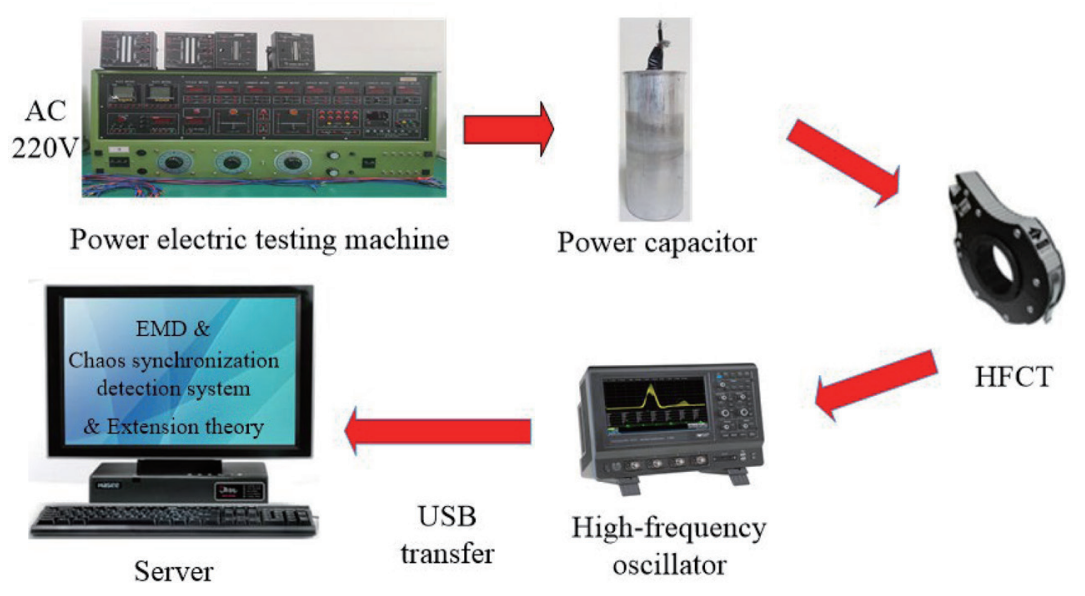

Fig. 3. (Color online) Flowchart of partial discharge low-voltage test.

\subsection{Partial discharge detection circuit}

The detection circuit is our original partial discharge signal detection circuit comprising a high-pass filter and a noninverting amplifier, as shown in Fig. 4.

\subsubsection{High-pass filter circuit}

Next, ENN was adopted to classify and recognize the chaotic eye coordinates. Finally, the recognition results were presented on the human-machine interface.

To increase the accuracy of the results in actual measurements, the low-frequency noise is filtered out by a high-pass filter. We use a passive filter as the high-pass filter, so as to capture the discharge signal with a longer oscillation effect and identify the extracted discharge signal clearly, as shown in Fig. 5. ${ }^{(19)}$

\subsubsection{Noninverting amplifier circuit}

To prevent severely high distortion and maintain the amplified high-frequency signal to some extent, this study used the current feedback amplifier (350 MHz bandwidth when $G=1$ and $35 \mathrm{MHz}$ bandwidth when $G=10$ ) with a low-distortion and high-frequency dynamic response and a negative feedback to stabilize the gain of the amplifier. The circuit is shown in Fig. 6.

\section{Proposed Fault Diagnosis Algorithms}

\subsection{EMD}

In the initial stage of the fault of the power panel board, the fault feature is not obvious. To enable the fault diagnosis system to identify the failure symptom more accurately, we propose 


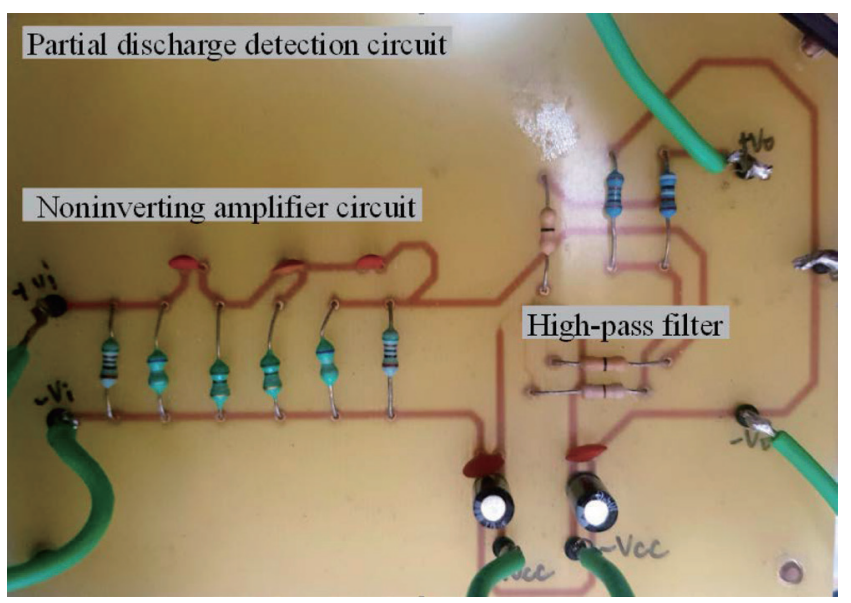

Fig. 4. (Color online) Partial discharge detection circuit.

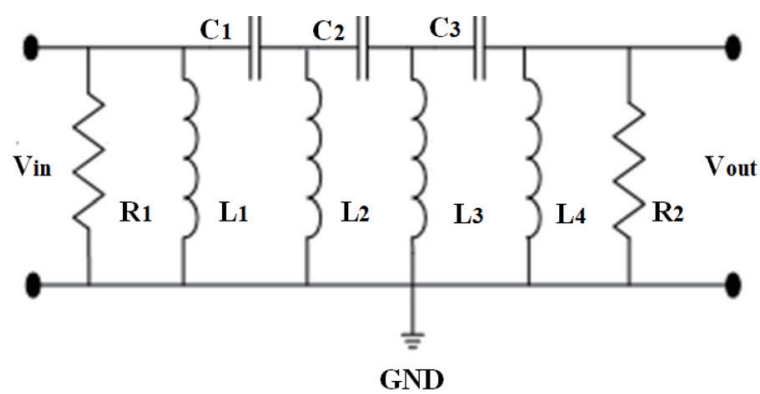

Fig. 5. High-pass filter circuit diagram.

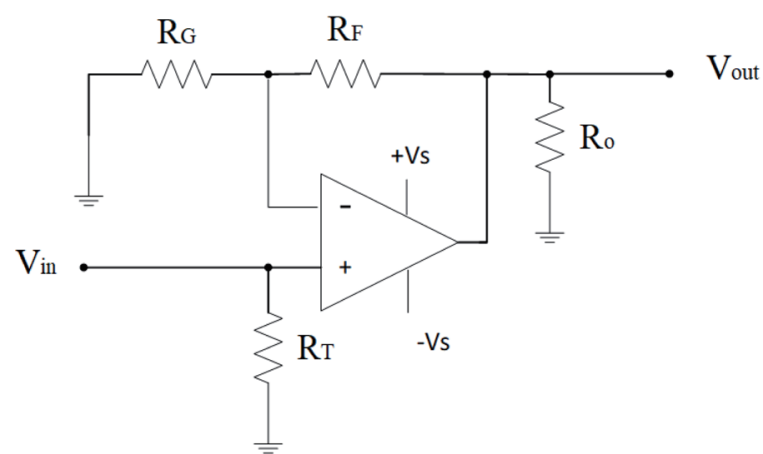

Fig. 6. Noninverting amplifier circuit diagram.

$\mathrm{EMD}^{(20)}$ as the preliminary signal conversion process because it can effectively separate the frequency components of the signal from the time curve in the form of the intrinsic mode function (IMF) and trace out the components of the original signal in different frequency bands by reconstruction. The important information implied in the original signal can be extracted during the course of decomposition. The basic equation is described below. If the time series signal is $x(t)$, all of the extreme points on $x(t)$ are determined, and then, all of the extreme points are combined by a curve. The upper and lower envelope curves of signal $x(t)$ are set as $u_{0}(t)$ and $v_{0}(t)$, and the average curve of the upper and lower envelopes is expressed as

$$
m_{0}(t)=\frac{1}{2}\left[u_{0}(t)+v_{0}(t)\right]
$$

When $m_{0}(t)$ is subtracted from $x(t), h_{1}(t)=x(t)-m_{0}(t)$. Generally, this does not satisfy the conditions for the IMF. $x_{1}(t)$ can be replaced by $h_{1}(t)$, and $u_{0}(t)$ and $v_{0}(t)$ can be calculated. The aforesaid process is repeated: 


$$
\left\{\begin{aligned}
m_{1}(t) & =\frac{1}{2}\left[u_{1}(t)+v_{1}(t)\right] \\
h_{2}(t) & =h_{1}(t)-m_{1}(t) \\
& \vdots \\
m_{k-1}(t) & =\frac{1}{2}\left[u_{k-1}(t)+v_{k-1}(t)\right] \\
h_{k}(t) & =h_{k-1}(t)-m_{k-1}(t)
\end{aligned}\right.
$$

In the above decomposition process, when a certain standard is reached after multiple repetitions, $h_{k}(t)$ becomes the first IMF of the original signal, which is set as $y_{1}(t)$. The rest of the signal is set as $r_{1}(t)$.

$$
\left\{\begin{array}{l}
y_{1}(t)=h_{k}(t) \\
r_{1}(t)=x(t)-y_{1}(t)
\end{array}\right.
$$

Here, $y_{1}(t)$ is the first component obtained by processing the original data, as well as the highest frequency component in the $x(t)$ of the original signal; $r_{1}(t)$ is the corresponding residual component. The $r_{1}(t)$ in the rest is used as the new original signal. This process is repeated, and EMD continues. Then, the second IMF $y_{2}(t)$ can be calculated. The process is repeated $n$ times until the signal is monotonic or its value is smaller than the present value, at which point the decomposition is complete. The IMF $y_{n}(t)$ and residual component $r_{n}(t)$ are obtained.

$$
\left\{\begin{array}{c}
r_{1}(t)-y_{2}(t)=r_{2}(t) \\
\vdots \\
r_{n-1}(t)-y_{n}(t)=r_{n}(t)
\end{array}\right.
$$

The important information in the fault signal can be extracted by repeated EMD and the noise interference can be eliminated.

\subsection{Chaos synchronization detection method}

The chaos theory is a chaos synchronization detection method proposed by the meteorologist Edward Norton Lorenz. ${ }^{(21)}$ This theory lays emphasis on the unsteady behavior of nonlinear dynamic systems. In a chaotic system, even if there are subtle changes in initial conditions, after a long-term amplification, there are huge differences in the results. Generally, the two chaotic systems are a master system and a slave system. When the initial values of the master and slave systems are different, the operation tracks of the two systems are also different, and the controller is installed at the back end of the slave system to track the master system. The controller enables the two different chaotic signals to have the same operation tracks after a period of time. This tracking system is called chaos synchronization, the basic equation of which is ${ }^{(22)}$ 


$$
\begin{gathered}
\lim _{t \rightarrow \infty}\left\|Y_{\text {slave }, i}(t)-X_{\text {master }, i}(t)\right\|=0 \\
i=1,2, \cdots, n,
\end{gathered}
$$

where $Y_{\text {slave }}$ is the slave system data and $X_{\text {master }}$ is the master system data. We use this theory to perform chaos synchronization detection for partial discharge signals. The master and slave chaotic systems are expressed as follows.

$$
\begin{gathered}
X_{\text {master }}=\left\{\begin{array}{c}
\dot{x}_{1}=f_{1}\left(x_{1}, x_{2}, \cdots, x_{n}\right) \\
\dot{x}_{2}=f_{2}\left(x_{1}, x_{2}, \cdots, x_{n}\right) \\
\vdots \\
\dot{x}_{n}=f_{n}\left(x_{1}, x_{2}, \cdots, x_{n}\right)
\end{array}\right. \\
Y_{\text {slave }}=\left\{\begin{array}{c}
\dot{y}_{1}=f_{1}\left(y_{1}, y_{2}, \cdots, y_{n}\right) \\
\dot{y}_{2}=f_{2}\left(y_{1}, y_{2}, \cdots, y_{n}\right) \\
\vdots \\
\dot{y}_{n}=f_{n}\left(y_{1}, y_{2}, \cdots, y_{n}\right)
\end{array}\right.
\end{gathered}
$$

Here, $f_{i}(i=1,2, \ldots, n)$ is a nonlinear function. The dynamic error is expressed as Eq. (8) by subtracting Eq. (7) from Eq. (6) and is expressed as Eq. (9).

$$
\begin{gathered}
\left\{\begin{array}{c}
e_{1}=y_{1}-x_{1} \\
e_{2}=y_{2}-x_{2} \\
\vdots \\
e_{n}=y_{n}-x_{n}
\end{array}\right. \\
\left\{\begin{array}{c}
\dot{e}_{1}=f_{1}\left(x_{1}, x_{2}, \ldots, x_{n}\right)-f_{1}\left(y_{1}, y_{2}, \ldots, y_{n}\right)=H_{1} \\
\dot{e}_{2}=f_{2}\left(x_{1}, x_{2}, \ldots, x_{n}\right)-f_{2}\left(y_{1}, y_{2}, \ldots, y_{n}\right)=H_{2} \\
\vdots \\
\dot{e}_{n}=f_{n}\left(x_{1}, x_{1}, \ldots, x_{n}\right)-f_{n}\left(y_{1}, y_{2}, \ldots, y_{n}\right)=H_{n}
\end{array}\right.
\end{gathered}
$$

We use two Lorenz chaotic systems to generate the chaos error distribution diagram, and the center of gravity is used as a feature to identify the occurrence of partial discharge signals. Equations (6) and (7) show the master and slave systems, respectively. The dynamic error state equation is calculated and expressed in matrix form as Eq. (9).

\subsection{ENN}

The ENN first proposed by one of the present authors (M.-H. Wang) is a pattern classification method based on concepts from the extension theory and neural networks. ${ }^{(23)}$ ENN allows that clustering problems have a range of features, supervised learning, continuous input, and discrete output. This ENN is a combination of the extension theory and a neural 
network algorithm. The extension theory provides a distance measurement for classification, while the neural network uses the features of parallel computation and learning capability. ENN has been successfully applied to power capacitor fault diagnosis. The schematic structure of ENN is displayed in Fig. 7. It contains both the input layer and the output layer. The nodes in the input layer obtain an input feature pattern and use a series of weight parameters to generate a map of the input pattern. In this network, there are two connection weights between the input nodes and an output node: one weight, $w_{k j}^{u}$, represents the upper bound and the other weight, $w_{k j}^{L}$, denotes the lower bound for this classic domain of features. This input pattern is further improved in the process characterized by the output layer. Only one output node in the output layer remains active to show a classification of the input pattern. The learning algorithm of ENN is discussed in the next section.

Several variables must be defined before learning. Let the training pattern set be $X=\left\{x_{1}, x_{2}, \ldots, x_{N p}\right\}$, where $N_{p}$ is the total number of training patterns. The $i$ th pattern is $X_{i}^{p}=\left\{x_{i 1}^{p}, x_{i 2}^{p}, \ldots, x_{i n}^{p}\right\}$, where $n$ is the total number of mathematical problems in engineering.

The number of feature patterns and the category of the $i$ th pattern is $p$. To assess the clustering performance, the number of errors is set as $N_{m}$, and the total error rate $E_{T}$ is defined as

$$
E_{T}=\frac{N_{m}}{N_{p}}
$$

The detailed supervised learning algorithm is given below.

Step 1. Set the connection weights between the input and output nodes on the basis of the range

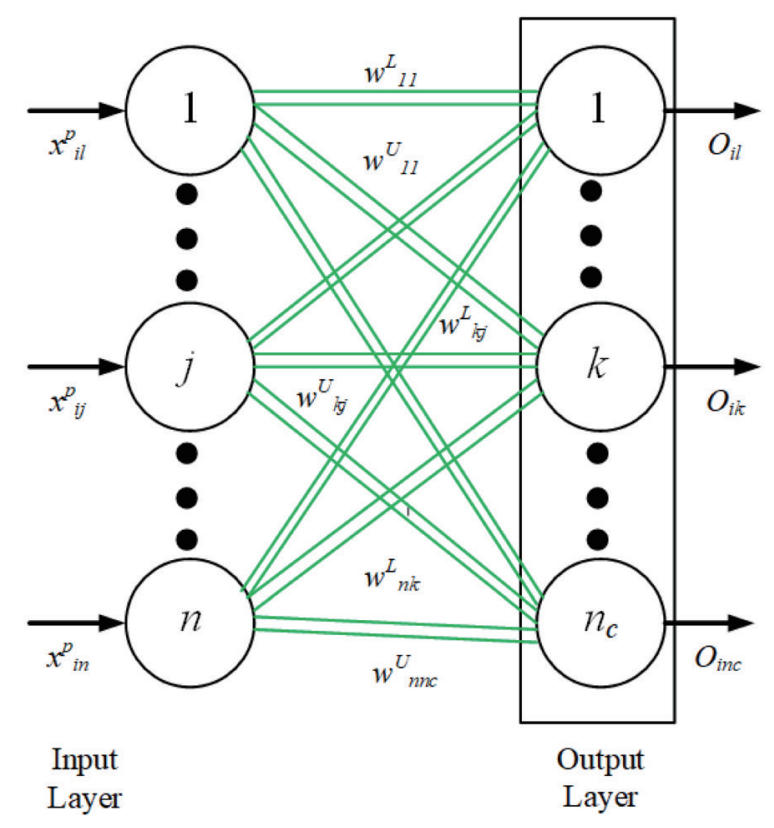

Fig. 7. (Color online) Structure of ENN. 
of classical domains that can be directly obtained from previous experience or decided from the training data.

Step 2. Calculate the weights of each feature median.

$$
\begin{gathered}
w_{k j}^{u}=\max _{i \in N p}\left\{x_{i j}^{k}\right\} w_{k j}^{u}=\min _{i \in N p}\left\{x_{i j}^{k}\right\} \\
\text { for } i=1,2, \ldots, N p ; j=1,2, \ldots, n ; k=1,2, \ldots, n_{c} \\
Z_{k}=\left\{z_{k 1}, z_{k 2}, \ldots, z_{k n}\right\} Z_{k j}=\frac{\left(w_{k j}^{L}+w_{k j}^{u}\right)}{2} \\
\text { for } k=1,2, \ldots, n_{c} ; j=1,2, \ldots, n
\end{gathered}
$$

Step 3. Read the $i$ th training pattern and cluster number $p$.

$$
X_{i}^{p}=\left\{x_{i 1}^{p}, x_{i 2}^{p}, \ldots, x_{i n}^{p}\right\}, p \in n_{c}
$$

Step 4. Use the extension distance to calculate the distance between the input pattern $X_{p i}$ and the $k$ th cluster.

$$
E D_{i k}=\sum_{j=1}^{n} \frac{\left|x_{i j}^{p}-z_{k j}\right|-\frac{w_{k j}^{L}-w_{k j}^{u}}{2}}{\left|\frac{w_{k j}^{L}-w_{k j}^{u}}{2}\right|} \text { for } k=1,2, \ldots, n_{c}
$$

Step 5. Find $k$, which is $E D_{i k}=\min \left\{E D_{i k}\right\}$. If $k=p$, then go to Step 7, otherwise go to Step 6 . Step 6. Update the weights of the $p$ th and $k$ th clusters.

$$
\begin{gathered}
\left\{\begin{array}{c}
w_{p j}^{L(\text { new })}=w_{p j}^{L(\text { old })}+\eta\left(x_{i j}-\frac{w_{p j}^{L(\text { old })}+w_{p j}^{U(\text { old })}}{2}\right) \\
w_{p j}^{U(\text { new })}=w_{p j}^{U(\text { old })}+\eta\left(x_{i j}-\frac{w_{p j}^{L(\text { old })}+w_{p j}^{U(\text { old })}}{2}\right)
\end{array}\right. \\
\left\{\begin{array}{c}
w_{m j}^{L(\text { new })}=w_{m j}^{L(\text { old })}+\eta\left(x_{i j}-\frac{w_{m j}^{L(\text { old })}+w_{m j}^{U(\text { old })}}{2}\right) \\
w_{m j}^{U(\text { new })}=w_{m j}^{U(\text { old })}+\eta\left(x_{i j}-\frac{w_{m j}^{L(\text { old })}+w_{m j}^{U(\text { old })}}{2}\right) \\
\text { for } j=1,2, \ldots, n_{c}
\end{array}\right.
\end{gathered}
$$

Here, $\eta$ is the learning rate, which is set at 0.1 in this paper. From this step, the purpose of the learning process is only to tune the weights of the $p$ th and $m$ th clusters.

Step 7. Repeat Steps 3 to 6. If all patterns have been classified, then the learning epoch is finished. 
Step 8. The process is finished if the clustering process has converged or the total error rate has arrived at a default value, otherwise return to Step 3.

\section{Actual Measurement and Result Analysis}

Figure 8 shows the power capacitor test platform. The power electric testing machine boosts the power capacitor until a partial discharge occurs. The HFCT as the sensor of the partial discharge signal is fixed to the ground terminal of the power capacitor. The detected signal change is transmitted via the Bayonet Neill-Concelman (BNC) connector to the filter amplifying circuit. The partial discharge signal of the power capacitor is analyzed by EMD and the chaos synchronization analysis method. The master system of the chaos synchronization detection system is set as 0 , which is regarded as the power capacitor baseline in the normal state.

Our experiment is based on three cycles of $220 \mathrm{~V} / 60 \mathrm{~Hz}$ AC. There are three aging types. Each data acquisition time is $50 \mathrm{~ms}$, the sampling frequency is $20 \mathrm{MS} / \mathrm{s}$, the number of sampling points is 5000, the discharge data are processed by EMD to obtain the IMF, and the center of gravity of the chaos scatter diagram is extracted as the eigenvalue of the fault detection by the Lorenz chaos synchronization detection method. The IMF of a normal capacitor is shown in Fig. 9. The IMF of an aged power capacitor is shown in Fig. 10.

The IMF (IMF1) is derived from the discharge waveform of the power capacitor after EMD, and the scatter diagram and the center of gravity (chaos eye) coordinate values are extracted by the Lorenz chaos synchronization detection method. The 3D chaotic scatter diagram, extracted from the normal capacitor by the Lorenz chaos synchronization detection method, is shown in Fig. 11. The 3D chaotic scatter diagram is decomposed to obtain a 2D plane chaotic scatter diagram and the center of gravity coordinate values, as shown in Fig. 12.

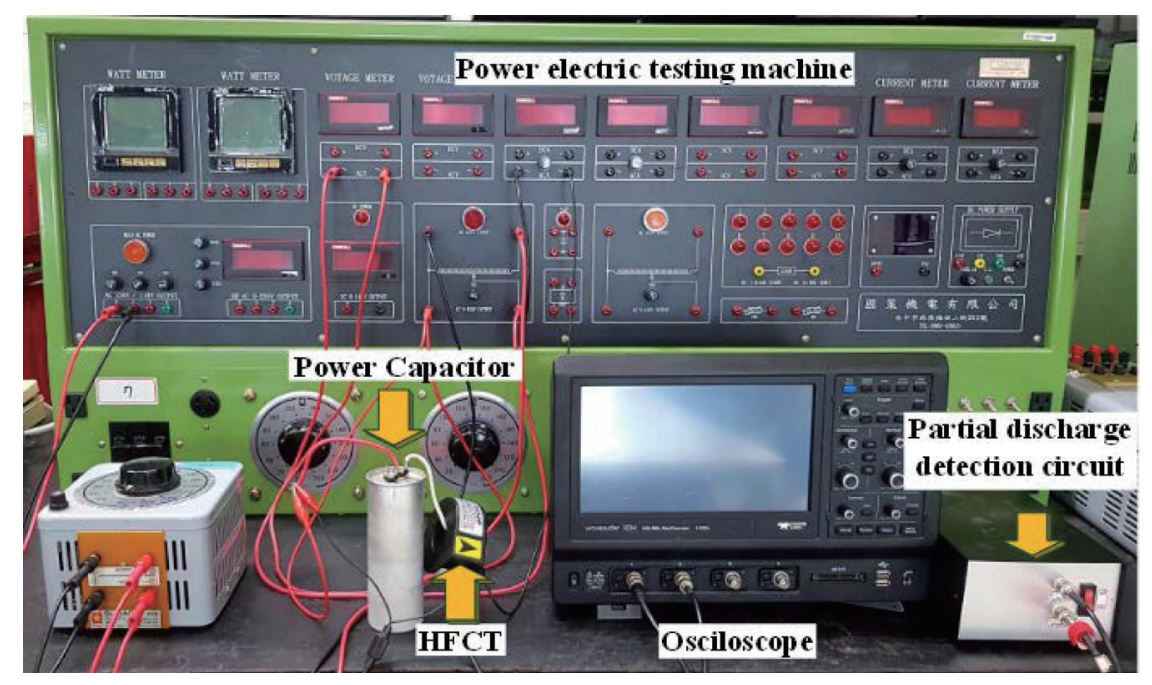

Fig. 8. (Color online) Power capacitor test platform. 


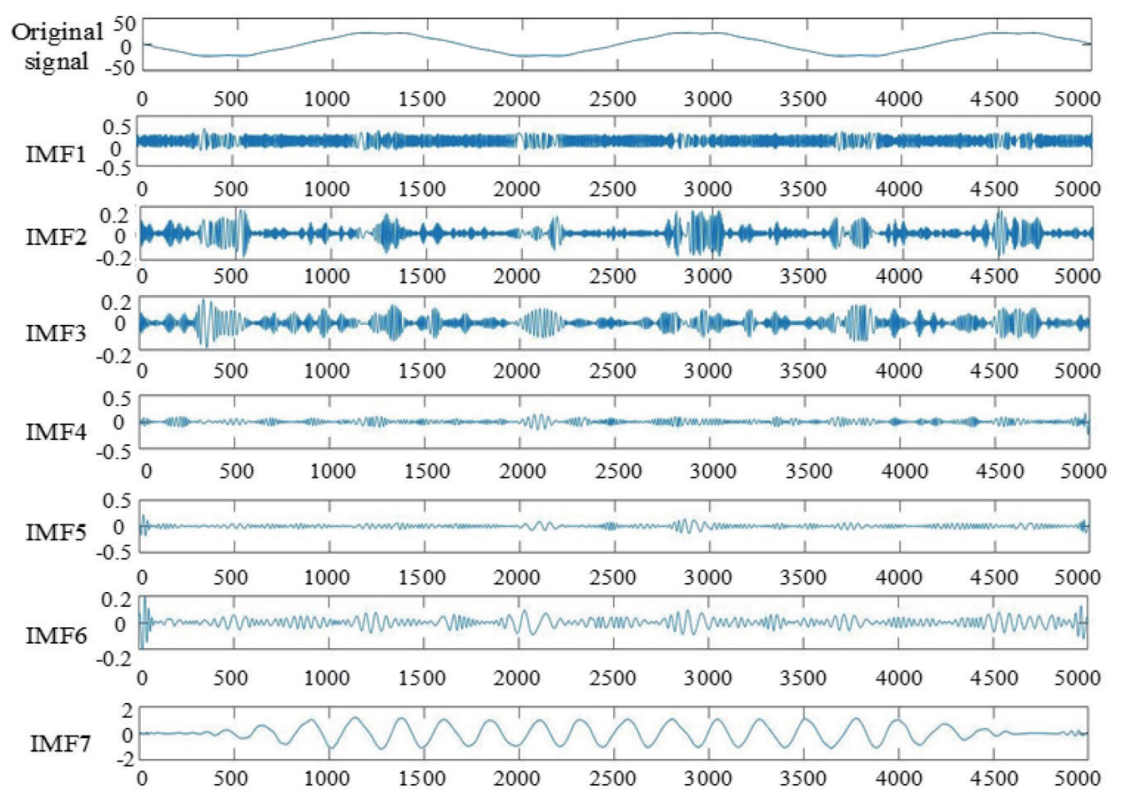

Fig. 9. (Color online) IMF of normal capacitor.

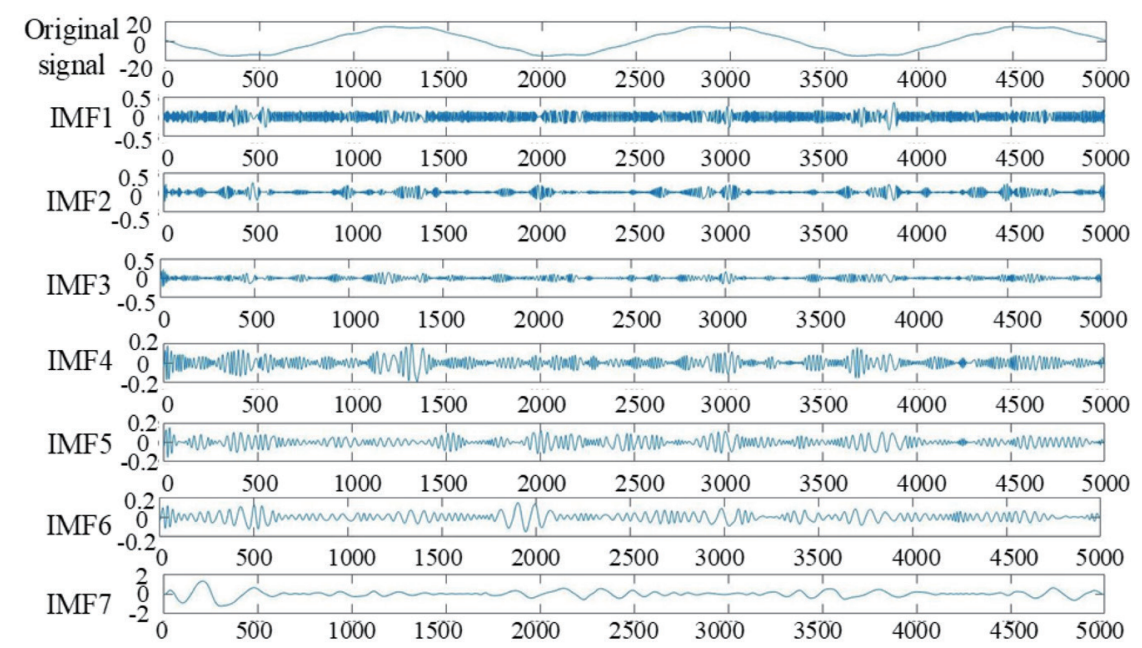

Fig. 10. (Color online) IMF of aged capacitor.

The 3D chaotic scatter diagram extracted from the aged capacitor by the Lorenz chaos synchronization detection method is shown in Fig. 13. By the above-described method, the 2D-plane chaotic scatter diagram and the center of gravity coordinate values are obtained after the 3D chaotic scatter diagram is decomposed, as shown in Fig. 14.

The barycentric coordinates of the $2 \mathrm{D}$ chaotic scatter diagram are combined with the subsequent extension theory, neural network, and ENN algorithms for calculation so as to judge the capacitor's state.

In this study, we used $\mathrm{C}_{++}$to compose the program of the ENN algorithm. The neural networks of other architectures were tested with two eigenvalue inputs and seven fault type 


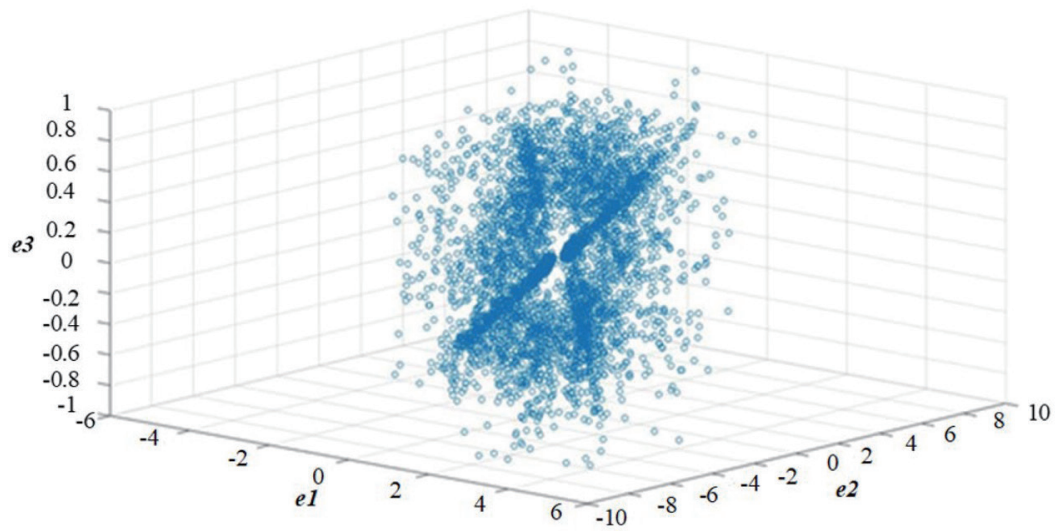

Fig. 11. (Color online) 3D chaotic scatter diagram of normal capacitor IMF1.

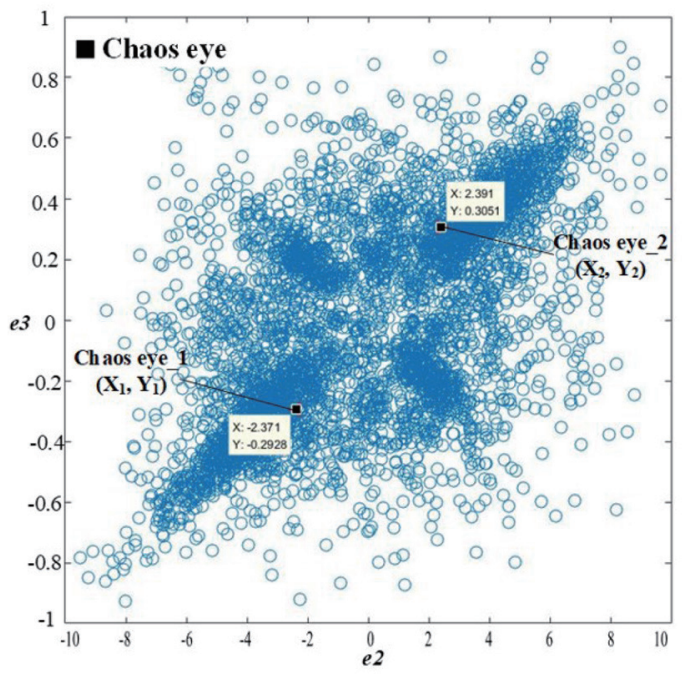

Fig. 12. (Color online) Chaotic scatter diagram and chaos eye of normal capacitor IMF1.

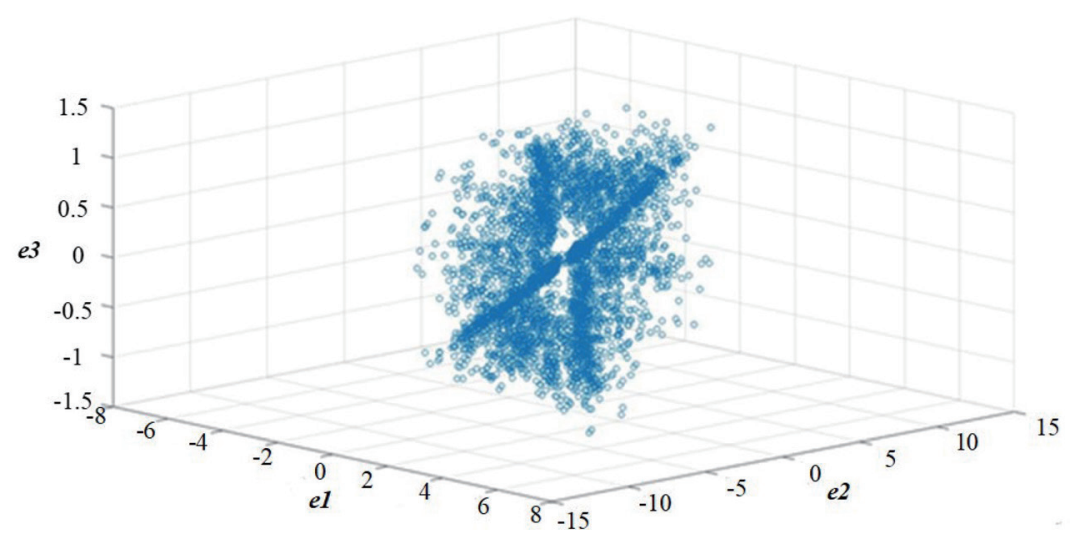

Fig. 13. (Color online) 3D chaotic scatter diagram of aged capacitor IMF1.

outputs. Only the hidden layer architecture setting was adjusted and tested with 10,12 , and 14 hidden layers. In terms of learning and overall recognition rates, the recognition accuracy 


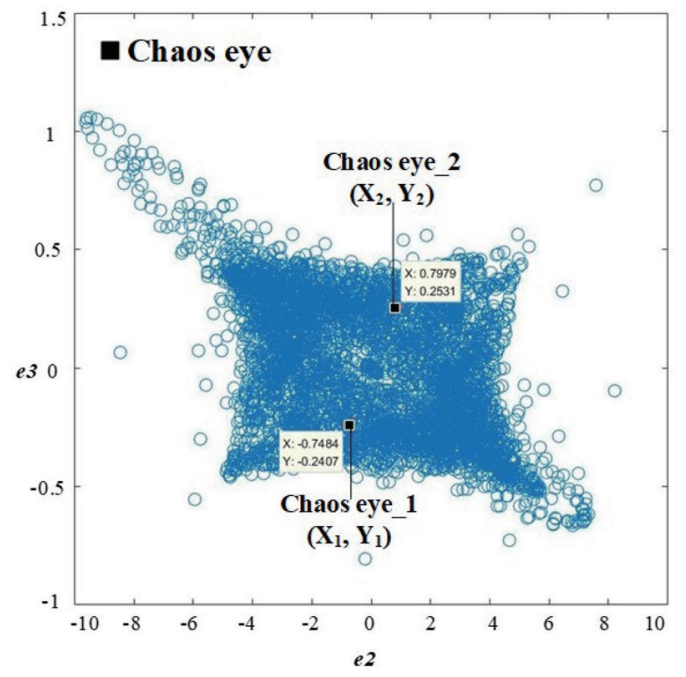

Fig. 14. (Color online) Chaotic scatter diagram and chaos eye of aged capacitor IMF1.

Table 2

Recognition results of ENN and other algorithms.

\begin{tabular}{lcccc}
\hline Algorithm & Training time & Learning rate (\%) & Accuracy rate (\%) & Ranking \\
\hline ENN & 1 & 100 & 95 & 1 \\
Multilayer neural network (4-10-7) & 10000 & 99 & 91 & 2 \\
Multilayer neural network (4-14-7) & 10000 & 99 & 90 & 3 \\
Multilayer neural network (4-12-7) & 10000 & 99 & 88 & 4 \\
Extension method & & & 84 & 5 \\
\hline
\end{tabular}

rate of 10 hidden layers was the highest at $91 \%$. The recognition result was compared with the extension method and ENN results, as shown in Table 2.

The recognition rate of ENN was the highest (95\%), the rate of the multilayer artificial neural network (4-10-7) was the second highest (91\%), and the rate of the extension theory was $84 \%$. The results show that ENN has a higher recognition accuracy than the other two algorithms, the maximum difference being $11 \%$.

\section{Conclusions}

We performed partial discharge detection by using an HFCT sensor, a high-pass filter, and a noninverting amplifier for power capacitors aged to different degrees (0-60\%). A chaotic scatter diagram was obtained from the data measured by EMD and the chaos synchronization detection method, and chaos eye coordinate values were extracted as the eigenvalues of fault diagnosis. Then, capacitor eigenvalues were analyzed by ENN for accurate fault category identification. The advantage of the proposed method is that the data can be compressed effectively to extract meaningful eigenvalues that increase the recognition accuracy of the algorithm. The findings show that the accuracy of the proposed method is as high as $95 \%$ compared with those of the multilayer neural network (ranging from 88 to 91\%) and extension method (84\%). 
The following are the characteristics of this study.

(1) The proposed EMD method has a simple and rapid calculation program. It can be combined with the chaos synchronization analysis method and ENN algorithm to further increase the power capacitor fault detection rate.

(2) The proposed chaotic scatter diagram can be used to provide power capacitor failure criteria. It can be combined with other algorithms for power capacitor fault diagnosis in the future.

(3) A human-machine interface graphic diagnostic program was implemented, and a realtime measurement and identification system was established. This method was integrated with EMD, the chaos synchronization detection method, and ENN for power capacitor fault diagnosis.

\section{Acknowledgments}

The authors gratefully acknowledge the financial support from the Ministry of Science and Technology of Taiwan, R.O.C., through its grant MOST 105-2221-E-167-014-MY3.

\section{References}

1 Z. Pooranian, J. H. Abawajy, P. Vinod, and M. Conti: Energies 11 (2018) 1. https://doi.org/10.3390/en11061348

2 C. Feng, J. Daozhuo, F. Yu, and G. Hongjie: IEEE Int. Conf. Intelligent Systems Design and Engineering Applications (2014) 841.

3 J. Pihera, P. Martínek, J. Klasna, B. Paslavský, R. Polanský, V. Mentlík, and P. Trnka: IEEE Conf. Electrical Insulation and Dielectric Phenomena (2009) 39.

4 M. R. Mauntz and J. Peuser: IEEE Conf. Diagnostics in Electrical Engineering (2016) 19.

5 J. M. Urtubi, A. Valls, and J. Sarda: IEEE Int. Conf. Exhibition on Electricity Distribution 1 (2014) 438.

6 P. Sharma, G. Kapoor, and S. Ali: IEEE Int. Conf. Computing, Power and Communication Technologies (2018) 754.

7 H. Jouybari-Moghaddam, T. Sidhu, I. Voloh, and M. Zadeh: IEEE 71st Annual Conf. Protective Relay Engineers (2018) 1.

8 J. Hannonen, J. Honkanen, J. Ström, T. Kärkkäinen, S. Räisänen, and P. Silventoinen: IEEE Trans. Ind. Appl. 52 (2016) 3224.

9 A. T. Carvalho, H. P. Amorim, C. F. C. Cunha, L. S. Ferreira, M. C. Cagido, and J. B. Borges: IEEE Electrical Insulation Conf. (2016) 511.

10 L. Cheng, Z. Li, and W. Liu: IEEE Int. Conf. Condition Monitoring and Diagnosis (2016) 282.

11 F. Ji, Q. Xu, Y. Zhu, H. M. Cheng, J. Bao, and J. Xu: IEEE Conf. Energy Internet and Energy System Integration (2018) 1.

12 M. H. Wang: IEE Proc. Gener. Transm. Distrib. 6 (2003) 679.

13 S. Laamami, M. Behamed, and L. Sbita: IEEE Int. Conf. Green Energy Conversion Systems (2017).

14 V. Divyashree and S. Sumathi: IEEE Int. Conf. Power and Advanced Control Engineering (ICPACE) (2015).

15 L. Ming: IEEE Int. Conf. Information and Financial Engineering (2010) 447.

16 M. H. Wang, S. D. Lu, and M. J. Hsieh: Measurement 129 (2019) 227.

17 T. Billard, F. Fresnet, M. Makarov, T. Lebey, P. Castelan, P. Bidan, and S. Dinculescu: IEEE Int. Conf. Solid Dielectr. (2013) 314.

18 A. Marinescu, I. Dumbrava, and L. Mandache: IEEE Int. Conf. High Voltage Engineering and Application (2018) 1.

19 F. Álvarez, E. Arcones, F. Garnacho, A. Ramírez, and J. Ortego: IEEE Int. Conf. High Voltage Engineering and Application (2018) 1.

20 X. Liang, M.Q Shi, H. Wu, X. P Wang, W. Zhou, and Q. Wang: Int. Conf. Modelling, Identification and Control (2017) 1003.

21 M. H Wang and Z. Y Lee: IEEE Int. Conf. Industrial Informatics (2016) 1247.

22 H. T. Yau and M. H. Wang: IET Renewable Power Generation 9 (2015) 6.

23 M. H. Wang and C. P. Hung: Proc. Int. Joint Conf. Neural Networks (2003) 779. 